\title{
EUV Line Intensities of Fe $\mathrm{X}$
}

\author{
P. R. YOUNG,${ }^{1}$ H. E. MASON, ${ }^{1}$ A. K. BHATIA, ${ }^{2}$ \\ G. A. DOSCHEK, ${ }^{3}$ AND R. J. THOMAS ${ }^{2}$ \\ ${ }^{1}$ Department of Applied Mathematics and Theoretical Physics, \\ Silver Street, Cambridge CB3 9EW, UK \\ ${ }^{2}$ Laboratory for Astronomy and Solar Physics, Code 680, \\ NASA-Goddard Space Flight Center, Greenbelt MD 20771, USA \\ ${ }^{3}$ E.O. Hulburt Center for Space Research, Naval Research Laboratory, \\ Washington DC 20375-5000, USA
}

The 4 configuration, distorted wave calculation of Bhatia \& Doschek (1995) (hereafter referred to as BD95), together with the ground transition calculation of Pelan \& Berrington (1995) are here used to predict the intensities of the Fe X EUV lines, which are then used to derive electron densities from several solar spectra, including the recent SERTS spectra.

\section{Introduction}

The identification of the red coronal line as being the ground transition of $\mathrm{Fe} X$ helped lead to the idea of the corona as a million degree plasma. The importance of Fe $\mathrm{X}$ today lies in it being formed over the temperature region $8-12 \times 10^{5} \mathrm{~K}$, bridging the different morphologies of the transition region and coronal plasmas.

The main EUV lines of $\mathrm{Fe} X$ are summarised in table 1: the $3 \mathrm{~s}^{2} 3 \mathrm{p}^{5}{ }^{2} \mathrm{P}-3 \mathrm{~s}^{2} 3 \mathrm{p}^{4} 3 \mathrm{~d}{ }^{2} \mathrm{P}$, ${ }^{2} \mathrm{D},{ }^{2} \mathrm{~S}$ transitions contribute over $80 \%$ of the power output of $\mathrm{Fe} \mathrm{X}$, giving rise to a complex of strong lines in the $170-190 \AA$ region; the $3 s^{2} 3 p^{4} 3 d{ }^{4} D_{5 / 2,7 / 2}$ levels are barely split and decay down to the $3 \mathrm{~s}^{2} 3 \mathrm{p}^{5}{ }^{2} \mathrm{P}_{3 / 2}$ level via electric dipole and magnetic quadrupole transitions, respectively, giving rise to a self-blended line observed at $257.26 \AA$.

\section{Atomic Data}

\subsection{Radiative Data and Energy Levels}

A 13 configuration model of $\mathrm{Fe} \mathrm{X}$ was used in the radiative code SUPERSTRUCTURE (Eissner et al. 1974), to produce transition probabilities and energy levels. The transition probabilities were found to be within $5 \%$ of those of Fawcett (1991). Observed energy levels from Corliss \& Sugar (1982) and Jupén et al. (1994), were used together with scaled SUPERSTRUCTURE energies for the remaining levels.

\subsection{Electron Excitation Data}

Mason (1994) assessed the (then) available electron collisional excitation data for $\mathrm{Fe} X$. Subsequently, a Distorted Wave calculation has been done by BD95 for 5 values of the incoming electron energy for a four configuration model of $\mathrm{Fe} \mathrm{X}$. This calculation is compared with two previous calculations in Figure 1 (left frame), where the reduced $\dagger$ collision strength is plotted against the reduced energy for the strong transition that gives rise to the $174.53 \AA$ line.

The high energy limit point at $E_{r}=1$ can be accurately determined for allowed transitions using oscillator strengths from, e.g., SUPERSTRUCTURE via the expression $4 \omega f / \Delta E$. This allows a crude comparison of the calculations-the BD95 results are

$\dagger$ see Burgess \& Tully (1992) for details 
TABLE 1. Important Fe $\mathrm{X}$ lines and their associated transitions. Note that, for $\mathrm{Fe} X,{ }^{2} \mathrm{P}_{3 / 2}$ is the ground level and ${ }^{2} \mathrm{P}_{1 / 2}$ is the first excited level.

\begin{aligned} & \multicolumn{2}{c}{ Upper Level } \\ & Configuration Term \\ & $3 \mathrm{~s}^{6} \mathrm{p}^{6}{ }^{2} \mathrm{~S}_{1 / 2} \\ & 3 \mathrm{~s}^{2} 3 \mathrm{p}^{4} 3 \mathrm{~d}{ }^{4} \mathrm{D}_{5 / 2,7 / 2} \\ &{ }^{2} \mathrm{D}_{5 / 2} \\ &{ }^{2} \mathrm{P}_{3 / 2} \\ &{ }^{2} \mathrm{~S}_{1 / 2} \\ &{ }^{2} \mathrm{D}_{3 / 2}\end{aligned}$

\begin{tabular}{rlc}
\multicolumn{2}{c}{ Lower Level } & Wavelength \\
Configuration & Term & $(\AA)$ \\
$3 \mathrm{~s}^{2} 3 \mathrm{p}^{5}$ & ${ }^{2} \mathrm{P}_{3 / 2}$ & 345.72 \\
& ${ }^{2} \mathrm{P}_{3 / 2}$ & 257.26 \\
& ${ }^{2} \mathrm{P}_{3 / 2}$ & 174.53 \\
& ${ }^{2} \mathrm{P}_{3 / 2}$ & 177.24 \\
& ${ }^{2} \mathrm{P}_{3 / 2}$ & 184.54 \\
& ${ }^{2} \mathrm{P}_{1 / 2}$ & 175.27
\end{tabular}

$\sim 20 \%$ more accurate than the Mann (1983) results due to the inclusion of the $3 \mathrm{~s}^{2} \mathrm{p}^{5} 3 \mathrm{~d}$ configuration, but a further $\sim 20 \%$ improvement is still possible. This can largely be achieved by including the $3 \mathrm{~s}^{2} 3 \mathrm{p}^{3} 3 \mathrm{~d}^{2}$ configuration (Nussbaumer, 1976).

\subsection{Resonance Structure in the Ground Transition}

BD95 did not include any resonance structure in their model, which is particularly important for the ground ${ }^{2} \mathrm{P}_{3 / 2}{ }^{2} \mathrm{P}_{1 / 2}$ transition. This calculation has been done separately by Pelan \& Berrington (1995), and the two are compared in figure 1 (right frame), together with the results of Mohan et al. (1994). Although this latter R-matrix calculation included some resonance structure, the fine-structure cross-section below the first excited state was not calculated (Pelan, private communication).
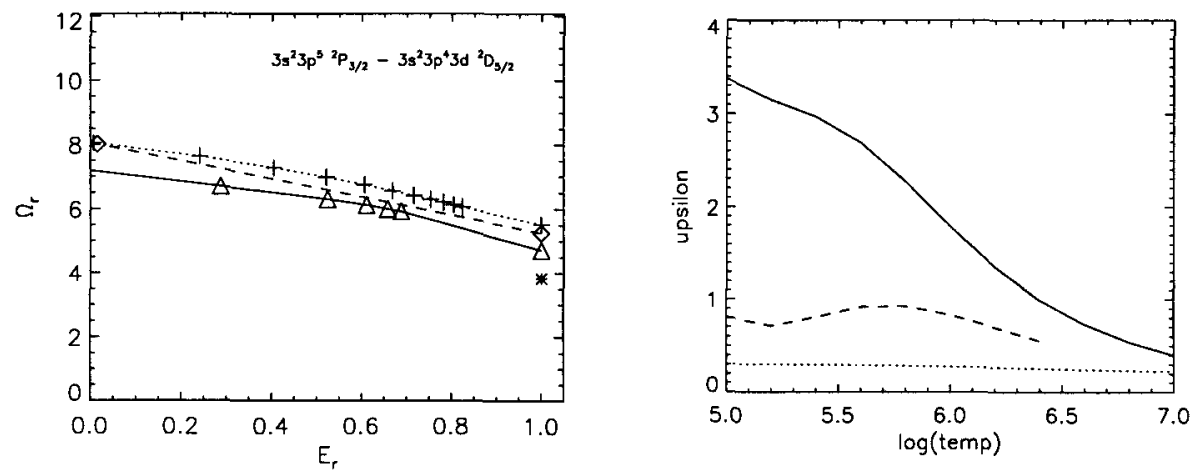

Figure 1. The left frame shows the reduced collision strength for the $\mathrm{Fe} X$ transition that gives rise to the $174.53 \AA$ line. The three sets of points represent the results of BD95 $(\Delta)$, Mason $1975(\diamond)$ and Mann $1983(+)$ * represents the accurate high energy limit point, and $E_{r}=0$ corresponds to the threshold energy of the transition. The right frame compares the thermally-averaged collision strengths for the ground ${ }^{2} \mathrm{P}_{3 / 2}-{ }^{2} \mathrm{P}_{1 / 2}$ transition obtained by: Pelan \& Berrington (-); Mohan et al. (- - ); Bhatia \& Doschek (.....).

\section{Density Diagnostics}

In the $170-190 \AA$ region, three density diagnostics are formed by taking the ratio of the $174.53,177.24$ and $184.54 \AA$ lines relative to the $175.27 \AA$ line. The new results for one 
TABLE 2. Intensity ratios for the $175.27 \AA$ line taken relative to the $174.53,177.24,184.54$ lines; the figure in brackets is the derived density. The observations used are: ${ }^{1}$ Malinovsky \& Heroux (1972); ${ }^{2}$ Behring et al. (1976); ${ }^{3}$ Dere et al. (1979); ${ }^{4}$ Drake et al. (1995). (This latter spectrum is of the star Procyon, observed by EUVE-the $184.54 \AA$ line was observed in a different bandpass and so the derived density is less certain.)

\begin{tabular}{|c|c|c|c|c|}
\hline Line & Full Disk Sun ${ }^{1}$ & Full Disk Sun ${ }^{2}$ & Solar Flare ${ }^{3}$ & Procyon $^{4}$ \\
\hline & $0.085(8.7)$ & $0.18(9.3)$ & $0.5(10.7)$ & $0.16(9.2)$ \\
\hline & $0.16(8.8)$ & $0.26(9.2)$ & $0.4(9.5)$ & $0.30(9.3)$ \\
\hline & $0.41(8.8)$ & $0.6(9.1)$ & n/a & 0.92 (9.5?) \\
\hline
\end{tabular}
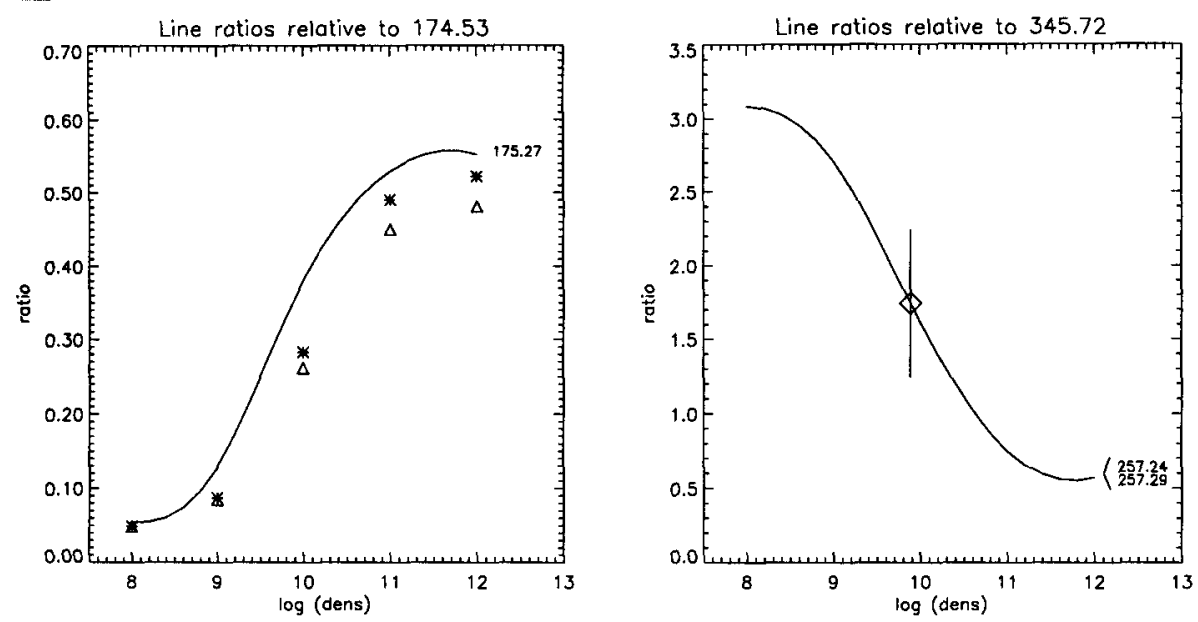

FIGURE 2. The left frame shows the new results presented here for the $175.27 / 174.53$ ratio (solid line). The $\Delta$ are the same results but with the BD95 collision strength for the ground transition, while the $*$ are the results of Brickhouse et al. (1994), who used the collision strengths of Mann for the transition shown. The right frame shows the 257.26/345.72 ratio with SERTS intensities and error bars marked on (note: theory splits the 257.26 blend into lines at $257.24, .29 \AA$ ).

of these ratios are presented in figure 2 (left frame), and used to derive the densities in table 2 for various spectra.

The 257.26/345.72 ratio is the only other useful density diagnostic in the EUV for Fe X and was observed by the Solar EUV Rocket Telescope and Spectrograph (SERTS) flight of 1989 .

\section{Results from SERTS}

\subsection{Densities Derived from $\mathrm{Fe} X$}

Figure 2 (right frame) shows the density obtained from the SERTS spectrum of Thomas \& Neupert (1994) using the Fe X 257.26 and $345.72 \AA$ lines. However this spectrum has been spatially-averaged over a region containing considerable inhomogeneities.

The variation of the SERTS emission over the slit of the spectrograph for the Fe $\mathrm{X}$ $257.26 \AA, 345.72 \AA$, and Fe XVI $335.40 \AA$ lines is shown in figure 3 . It can be seen that the subflare (seen clearly near the centre of the slit in the Fe XVI line), gives different 

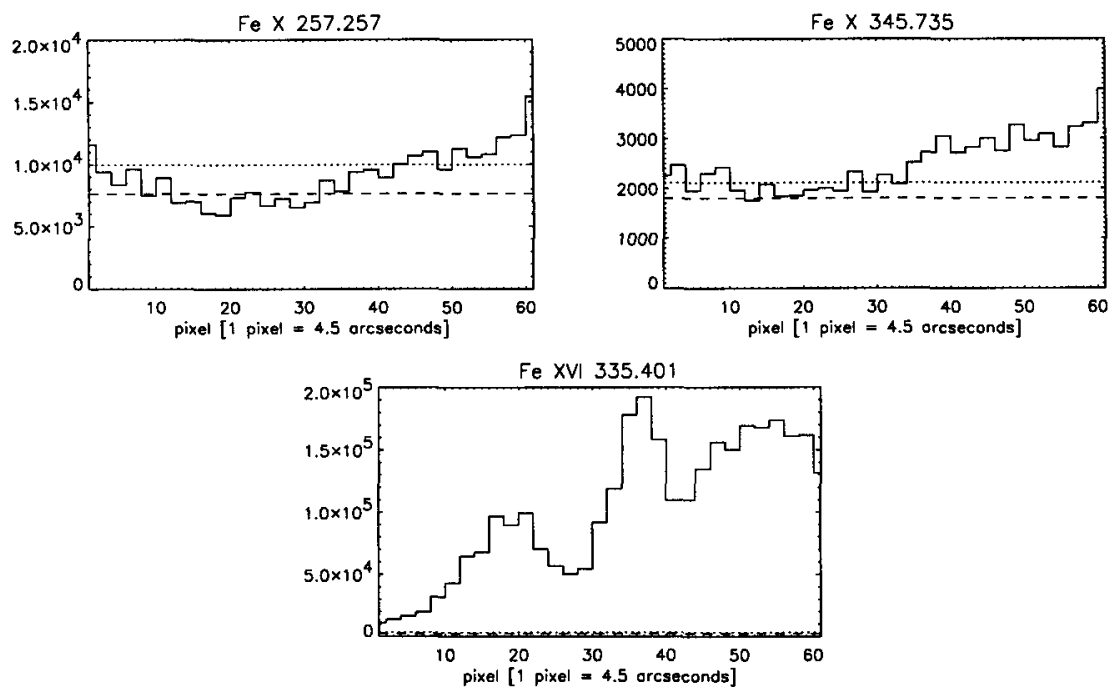

Figure 3. Spatial variation across the SERTS slit of Fe $X 257.26 \AA$ and $345.72 \AA$ (seen at $345.735 \AA$ by SERTS), and Fe XVI 335.40 $\AA$. The dashed line represents the average background, while the dotted line represents the $1 \sigma$ variation of the background.

TABLE 3. Densities derived from the SERTS spectrum for various ions formed around $10^{6} \mathrm{~K}$. The collisional data for Mg VIII and Si X are from Zhang et al. (1994) and this, together with the radiative data were supplied by K. P. Dere.

$\begin{array}{ccccc}\text { Ion } & T_{\max } & \text { Ratio } & \text { Density } & \text { Data Source } \\ \text { Mg VIII } & 5.9 & 436.73 / 430.45 & 5.0 \times 10^{8} ? & \text { present } \\ \text { Si VIII } & 5.9 & 276.85 / 319.84 & 2.5 \times 10^{10} & \text { Dwivedi }(1994) \\ \text { Fe X } & 6.0 & 257.26 / 345.72 & 8.9 \times 10^{9} & \text { present } \\ \text { Si IX } & 6.0 & 349.87 / 345.13 & \gtrsim 10^{10} & \text { Mason \& Bhatia (1978) } \\ \text { Si X } & 6.1 & \mathbf{3 5 6 . 0 3 / 3 4 7 . 4 1} & 1.3 \times 10^{9} ? & \text { present }\end{array}$

contributions to the two $\mathrm{Fe} X$ lines. One can estimate the subflare 257.26/345.72 ratio as $\approx 1.2$ and the active region loops (to the right of the subflare) ratio as $\approx 2.5$, leading to densities $\approx 2.5 \times 10^{10}$ and $\approx 2.0 \times 10^{9}$, respectively.

\subsection{Comparison with Other Ions}

An important study for both solar and atomic physics is to check if ions with similar temperatures of peak abundance $\left(T_{\max }\right)$ give consistent results for the electron density. For Fe X, with $T_{\max }$ around $1 \times 10^{6} \mathrm{~K}$, we have Si VIII-X and Mg VIII, all with useful density diagnostics lying within the SERTS wavelength range.

The densities predicted by these diagnostics are shown in table 3 where one can see a fair degree of scatter. However the Mg VIII ratio is only sensitive to densities $\lesssim 10^{9}$, and the error bars are large enough to make this density estimate highly uncertain. The Si X ratio involves the $347.41 \AA$ line which was marred by a plate flaw (Thomas \& Neupert, 1994), again making the density estimate uncertain. 
TABLE 4. Wavelength bands in $\AA$ covered by CDS

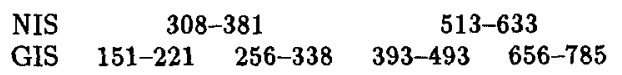

\section{Future Observations}

The SERTS rocket will be flown again in the spring of 1995 , with a new multilayercoated grating tuned to enhance the spectral region between $170-220 \AA$. This should allow the full set of $\mathrm{Fe} X$ diagnostics to be observed in that wavelength range for the first time ever from individual solar features.

In addition, the Solar and Heliospheric Observatory (SOHO), to be launched in October 1995, will contain several instruments including the Coronal Diagnostic Spectrometer (CDS) which will cover the EUV region of the solar spectrum. CDS's two spectrometers (the grazing incidence, GIS, and the normal incidence, NIS) will cover most of the $150-800 \AA$ region in the bands shown in table 4.

As can be seen, the $170-190 \AA$ lines will all be observed, together with the 257.26 blend and the 345.72 line. However, these latter two lines are covered separately by the GIS and NIS, respectively, and so the ratio will be subject to calibration uncertainties.

The 345.72 and 174.53 lines may be used to help cross-calibrate the GIS and NIS since the $345.72 / 174.53$ ratio is relatively density and temperature insensitive over $\log T=$ $6.0 \pm 0.15$ and $\log N_{e} \sim 8-10$, with values $0.02-0.05$.

PRY and HEM acknowledge the support of EPSRC and PPARC, respectively.

\section{REFERENCES}

Behring, W. E., Cohen, L., Feldman, U., \& DoscheK, G. A. 1976, ApJ, 203, 521

Bhatia, A. K. \& DoscheK, G. A. 1995, ADNDT, in press

Brickhouse, N. S., RAYMond, J. C., \& SMITH, B. W. 1995, ApJS, in press

Burgess, A. \& Tully, J. A. 1992, A\&A, 254, 436

Corliss, C. \& Sugar, J. 1982, J. Phys. Chem. Ref. Data, 11, 1

Dere, K. P., Mason, H. E., Widing, K. G., \& Bhatia, A. K. 1979, ApJS, 40, 341

Drake, J. J., Laming, J. M., \& Widing, K. G. 1995, ApJ, 443, 393

DwIVEdr, B. N. 1994, Sol. Phys., 153, 199

Eissner, W., Jones, M., \& Nussbaumer, H. 1974, Comp. Phys. Comm., 8, 270

FAWCETT, B. C. 1991, ADNDT, 47, 319

JuPÉN, C., ISLER, R. C., \& TrABËrt, E. 1994, MNRAS, 264, 627

MaLINOVSKY, M. \& HerouX, L. 1973, ApJ, 181, 1009

MANN, J. B. 1983, ADNDT, 29, 407

MASON, H. E. 1975, MNRAS, 170, 651

MASON, H. E. 1994, ADNDT, 57, 305

Mason, H. E. \& Bhatia, A. K. 1978, MNRAS, 184, 423

Mohan, M., Hibbert, A., \& Kingston, A. E. 1994, ApJ, 434, 389

NussBaumer, H. 1976, A\&A, 48, 93

Pelan, J., \& Berrington, K. A. 1995, A\&AS, in press

Thomas, R. J. \& Neupert, W. M. 1994, ApJS, 91, 461

Zhang, H. L., Graziani, M., \& Pradhan, A. K. 1994, A\&A, 283, 319 